\title{
LOTSIZING AND SCHEDULING WITH SEQUENCE-DEPENDENT SETUPS VIA BATCH SEQUENCING
}

\author{
Carsten Jordan, Christian-Albrechts Universität Kiel \\ Andreas Drexl, Christian-Albrechts Universität Kiel
}

A single stage multi item capacitated dynamic lotsizing and scheduling model with setup-times and costs is formulated and solved as a batch sequencing model (BSM). We consider the Discrete Lotsizing and Scheduling Problem with Setup Times (DLSPST, cf.[1]) which determines simultaneously the size and sequence of lots. We investigate the advantages and drawbacks of the BSM with respect to the DLSPST under modelling aspects and concerning solution algorithms.

We present a BSM (cf. [2], [3]), where lots consist of an integral number of demands, which are batched together. Demands are interpreted as jobs with a deadline, a certain processing time and earliness penalties. Analogously to the items the set of jobs is partitioned into jobclasses, and setups occur only between different jobclasses (=items). Earliness penalties of a job equal processing times multiplied with holding costs of its jobclass.

The corresponding scheduling problem now consists of scheduling the jobs between zero and their deadline on a single machine taking into account the (sequence-dependent) setup-times between jobclasses. Early completion of a job is weighted with its earliness-costs and setup-costs occur when switching from one jobclass (=item) to another. The objective is to minimize the sum of earliness- and setup-costs.

The relationship between both models is illustrated by two theorems, which are easily proven with exchange arguments:

Theorem 1 (cf. [ ] ]): If there exists a feasible solution for the DLSPST (where a demand may be splitted into different lots), then there exists a feasible solution for the BSM (with unsplitted demand), too.

Theorem 2: In the case of identical holding costs for all items (w.l.o.g. $h_{i}=1 \forall i$, so that earliness costs equal processing times) each schedule with splitted demand can be transformed into a schedule with unsplitted demand with less or equal costs. Thus there is also an optimal schedule with unsplitted demand.

The BSM is solved with an enumerative approach and compared with the dual ascent and column generation approach for the DLSPST. Therefore the DLSPST instances of [1], where holding costs are identical for all items are transformed into BSM instances. Computational results show, that the first approach outperforms the second one even for the special case of sequence-independent setups though the BSM accommodates also sequence-dependent setups.

\section{References}

[1] Cattrysse, D., M. Salomon, R. Kuik and L. N. van Wassenhove, 1993. A Dual Ascent and Column Generation Heuristic for the Discrete Lotsizing and Scheduling Problem with Setup Times, Management Science, Vol. 39, Nr. 4.

[2] Unal, A., and A. S. Kiran, 1992. Batch Sequencing, IIE Transactions, Vol. 24, pp.73-83.

[3] Bruno, J. and P. Downey, 1978. Complexity of task sequencing with deadlines, setup-times and changeover costs, SIAM Journal on Computing, Vol.7, pp. 393-404. 